\title{
TRAIL and immunity: more than a license to kill tumor cells
}

\author{
N Corazza ${ }^{1}$, G Brumattii ${ }^{1}$, C Schaer ${ }^{1}$, I Cima ${ }^{1}$, C Wasem ${ }^{1}$ and \\ T Brunner, ${ }^{*}$
}

The continuously growing family of structurally related proteins of the tumor necrosis factor (TNF) family has received enormous scientific attention due to their important role in the regulation of cell activation, survival, death and many other physiological and pathophysiological events (reviewed in Locksley et al. ${ }^{1}$ ). In this News and Commentary, we will focus on the only recently recognized and appreciated role of TNF-related apoptosis-inducing ligand (TRAIL) in regulating various physiological and pathophysiological processes involving immune cells.

\section{TRAIL-induced apoptosis in immune cells}

There is accumulating evidence that TRAIL-induced apoptosis is not limited to transformed cells, but may also be induced in primary cells. It is likely that this observation has been missed previously since TRAIL sensitivity in primary cells may be under stringent control and only be activated under certain circumstances. For example, primary mouse plasma cells generated in a T-cell-dependent immune response become sensitive to TRAIL-induced apoptosis. By contrast, resting and activated $B$ cells are resistant to TRAIL-mediated killing despite comparable expression of TRAIL receptors. ${ }^{2}$ The selective sensitivity of plasma cells correlates with decreased expression of CD40 and reduced activation of $\mathrm{NF} \kappa \mathrm{B}$, thus leading most likely to lower levels of antiapoptotic molecules. The in vivo relevance of this TRAIL-based elimination of plasma cells is uncertain, since no accumulation of plasma cells has been observed so far in the absence of TRAIL, although neutralization of TRAIL in vivo leads to increased antibody production. ${ }^{3}$ The observation proves, however, that depending on the activation and differentiation status also primary cells can undergo TRAIL-induced apoptosis.

At least two other types of immune cells also appear to be regulated by TRAIL. Dendritic cells (DC) play an important role in the initiation of an immune response by presenting antigens to antigen-specific T cells. Also, immature DC have been described to be sensitive to TRAIL-induced apoptosis. ${ }^{4}$ Recent studies indicated a role for NK cells in the regulation of
DC via TRAIL-induced apoptosis. The in vivo importance of this regulatory pathway has been confirmed by the elimination of NK cells and/or neutralization of TRAIL function during immunization with immature DC, loaded with non-self or tumor antigens. ${ }^{5}$ The reduced elimination of antigen-presenting DC by TRAIL-induced apoptosis resulted in a significantly enhanced T-cell response. These data provide evidence for the role of TRAIL in immune regulation and indicate the potential of TRAIL neutralization as an adjuvant during vaccination to improve T-cell responses to tumor antigens. Neutralization of TRAIL must remain, however, transient since TRAIL expressed by cytotoxic lymphocytes appears to be crucial for tumor control.

Neutrophilic granulocytes have a short lifespan and their survival is strongly regulated by a differentiation-induced program of apoptosis induction. Death receptors have been previously shown to accelerate neutrophil apoptosis, in particular Fas, and might thus contribute to the resolution of tissue inflammation. ${ }^{6}$ In a recent publication, Renshaw et al. ${ }^{7}$ demonstrated that neutrophil apoptosis is also accelerated by leucine zipper-tagged TRAIL, which may mimic transmembrane TRAIL on a cell surface. This susceptibility of neutrophils to TRAIL-mediated apoptosis suggests a role for TRAIL in the regulation of inflammatory reactions and may provide a potential mechanism of neutrophil clearance at sites of inflammation to prevent excessive tissue damage. Surprisingly, human neutrophils not only express TRAIL receptors but also the ligand, ${ }^{8}$ which is capable of mediating apoptosis in tumor cells. Thus, neutrophil-expressed TRAIL may contribute to tumor surveillance in vivo. Given that TRAIL and TRAIL receptors are co-expressed on differentiated neutrophils, it is tempting to speculate that TRAIL/TRAIL receptor interaction may be responsible for the substantial cell death observed in differentiated aged neutrophils and the short halflife of this cell population. However, ex vivo neutralization of TRAIL does not lead to increased survival of neutrophils, ${ }^{7}$ arguing against a TRAIL/TRAIL receptor-dependent suicide mechanism, though it is possible that ligand/receptor interaction has taken place already in vivo. All together, these different studies emphasize the role of TRAIL in modulating immune responses through induction of apoptosis in several subsets of immune cells.

\section{TRAIL and thymic negative selection}

Thymocyte apoptosis represents an important mechanism for the maintenance of self-tolerance and shaping of the T-cell receptor (TCR) repertoire through elimination of autoreactive or nonreactive thymocytes. Simon et al. ${ }^{9}$ have found that human thymocytes express TRAIL-R1 and -R2, which become further upregulated upon TCR stimulation. Most notably, the TRAIL receptors appear to be functional since considerable cell death was observed upon receptor ligation. 
Given that also TRAIL expression has been observed in the thymus, ${ }^{10,11}$ it has been tempting to speculate whether TRAIL/TRAIL receptor interaction might be (one of) the underlying mechanism(s) of thymic negative selection, that is, the induction of apoptosis in autoreactive thymocytes upon TCR ligation. Indeed, recently Lamhamedi-Cherradi et al. ${ }^{12}$ have described that $\mathrm{CD} 4{ }^{+} \mathrm{CD} 8^{+}$thymocytes from TRAILdeficient mice are more resistant to TCR-mediated apoptosis than those from wild-type mice. They also found that soluble TRAIL-R2 is able to block activation-induced cell death in thymocytes. This would suggest that high-affinity TCR ligation causes the expression of TRAIL receptor and/or TRAIL on double-positive thymocytes and subsequent interaction leads to apoptosis, that is, as has been described previously for Fas and FasL in activation-induced cell death in mature T cells. ${ }^{13}$ Since thymic negative selection should be impaired in TRAILdeficient mice, it is predicted that the uncontrolled escape of autoreactive $\mathrm{T}$ cells into the peripheral immune system leads to the development of spontaneous autoimmune diseases. Interestingly, however, Lamhamedi-Cherradi et al. ${ }^{12}$ could demonstrate that TRAIL-deficient mice show an increased susceptibility to experimentally induced autoimmune disease, but so far any evidence is lacking that TRAIL-deficient mice would develop similar autoimmune disorders as $I p r$ and gld mice. Thus, the idea of Lamhamedi-Cherradi et al. has been recently challenged by Cretney et al., ${ }^{14}$ who have not been able to reproduce the observation that negative selection is impaired in TRAIL-deficient mice. Another important aspect that argues against a direct role of TRAIL-TRAIL receptor interaction in central deletion is the fact that the adaptor protein FADD is not essential for thymic negative selection. ${ }^{15}$ These differences are difficult to reconcile. Our own recent results may, however, shed a new light on the role of TRAIL in thymocyte apoptosis and may explain some of the controversies. Using identical TRAIL-deficient mice, we observed reduced apoptosis of double-positive thymocytes upon TCR activation in vivo and in vitro, thus confirming some of the results of Lamhamedi-Cherradi et al. In addition, though, we have also found reduced thymocyte apoptosis upon treatment with UV or $\gamma$-irradiation and glucocorticoids, but not with antiFas antibody. ${ }^{16}$ TCR-, UV irradiation-, $\gamma$-irradiation- and glucocorticoid-induced thymocyte apoptosis proceeds via the mitochondria, that is, is blockable by transgenic overexpression of $\mathrm{Bcl}-2$, whereas Fas-induced apoptosis is normal due to type I signaling, without further amplification of the signal via Bid cleavage and cytochrome $C$ release. ${ }^{17}$ This differential induction of apoptosis would thus suggest that the regulation of the mitochondrial apoptosis pathway is different in TRAIL-deficient versus wild-type thymocytes. Indeed, we have found that TRAIL-deficient thymocytes show a reduced activation-induced expression of Bim (protein and mRNA), ${ }^{16}$ a proapoptotic member of the Bcl-2 family already previously implicated in thymocyte apoptosis. ${ }^{18}$ TRAIL receptor activation can cause the activation of Jun kinase (JNK) via the adaptor protein RIP in the absence of FADD. ${ }^{19} \mathrm{JNK}$ in turn can regulate Bim expression on a transcriptional and post-transcriptional level. ${ }^{20,21}$ We thus propose that in the thymus TRAIL is a response modifier for mitochondrial apoptosis pathways rather than a direct mechanism of thymic negative selection ${ }^{16}$ (Figure 1a).

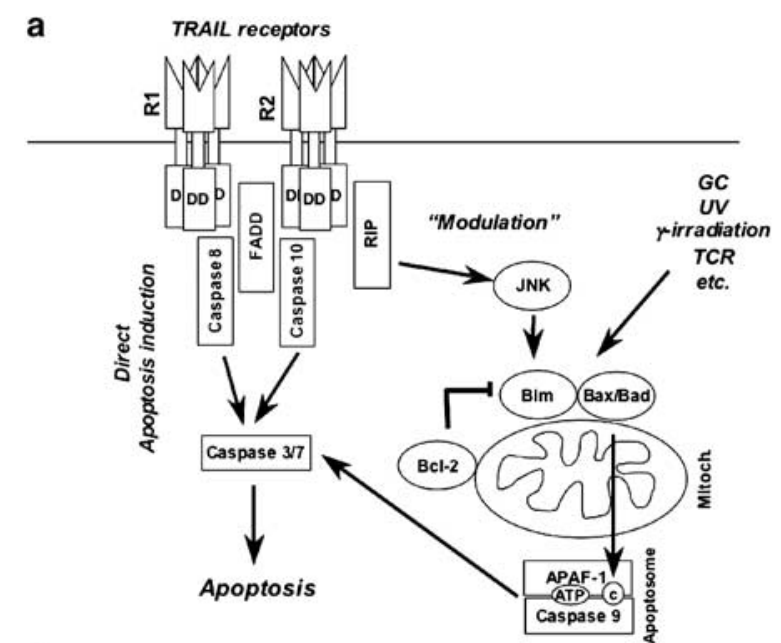

b

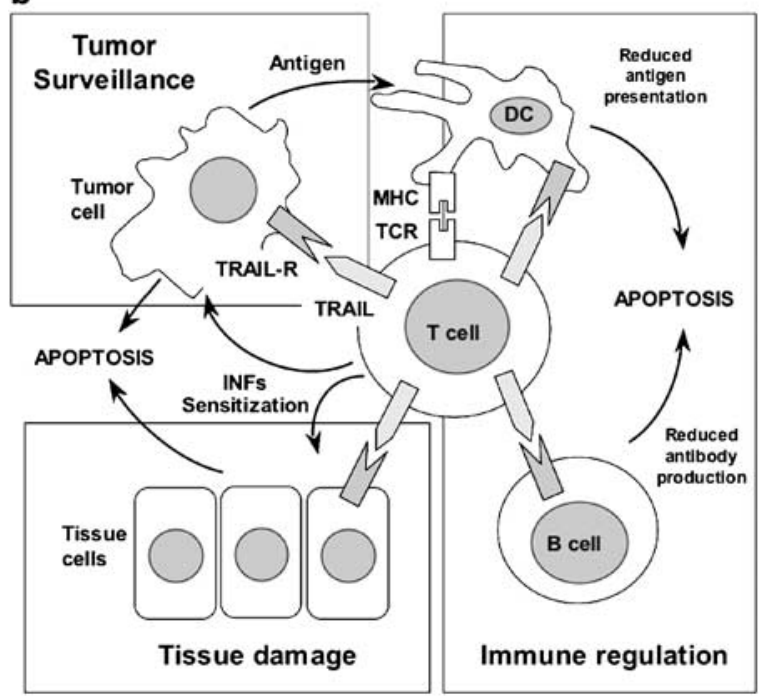

Figure 1 (a) Possible mechanisms of apoptosis induction and regulation by TRAIL. TRAIL may either directly induce apoptosis via TRAIL receptor 1 or 2 , FADD recruitment and caspase activation, or modulate the mitochondrial apoptosis pathway through activation of JNK and induction/stabilization of the proapoptotic Bcl-2 homolog Bim. Thereby, TRAIL signaling may enhance apoptosis induction by glucocorticoids (GC), UV and $\gamma$-irradiation, or TCR signaling. (b) Proposed role of TRAIL in tumor surveillance, tissue destruction and immune regulation. Tumor antigen may be presented by DC to $T$ cells, and $T$ cells may kill tumor cells via TRAIL/TRAIL-R interaction. Activated T cells, may, however, also induce apoptosis in nontransformed tissue cells, leading to tissue damage. Similarly, TRAIL-induced apoptosis of DC leads to reduced antigen presentation and reduced T-cell activation, TRAIL-induced apoptosis of B cells and plasma cells to reduced antibody secretion. Interferons (IFNs) and other proinflammatory cytokines may sensitize tissue cells and tumor cells for TRAILinduced apoptosis. MHC, major histocompatibility complex; TCR, T-cell receptor

\section{Role of TRAIL in immune regulation, immunopathologies and autoimmune diseases}

The data discussed above clearly point out that (i) TRAIL can also induce apoptosis or at least modulate apoptosis sensitivity in primary cells, and (ii) that TRAIL might be 
involved in various physiological and pathophysiological processes. In addition, there is also an emerging role for TRAIL in various processes involving the activation and regulation of immune cells. A common theme of all these studies is the observation that TRAIL activity appears to have both a destructive as well as a regulatory potential. Thus, TRAIL seems to be involved in tissue damage, but also in the tight control of immune cells to prevent any harm of tissue cells. Again, parallels to the FasL/Fas system become evident, where FasL has been demonstrated to be responsible for excessive tissue cell death during various pathological processes, and at the same time represents a safeguard mechanism by inducing T-cell apoptosis and thus preventing excessive T-cell activation and cytotoxic effector functions (reviewed in Brunner et al. ${ }^{22}$ ). For example, FasL is also expressed by the eye, where it maintains an immunologically privileged environment by inducing apoptosis in infiltrating leukocytes. ${ }^{23}$ Similarly, under physiological condition, TRAIL is also constitutively expressed in different organs, suggesting that it could be implicated in the immune surveillance. ${ }^{24}$ In agreement with this notion, it has been observed that some tumor cells not only express FasL but also TRAIL, which may also contribute to immune escape. ${ }^{25}$ Likewise, TRAIL expression in the human placenta has been associated with the maintenance of immune privilege. ${ }^{26}$ Interestingly, however, while Fas (Ipr) and FasL ( $g / d)$ mutant mice show clear symptoms of uncontrolled immune cell activation, defects in cellular homeostasis, reduced apoptosis and development of autoimmune disease, ${ }^{27}$ none of these signs is obvious in the TRAIL-deficient mouse, at least not at the first glance. ${ }^{28,29}$ Yet, a more detailed analysis revealed that lack of TRAIL predisposes to increased susceptibility to the development of autoimmune diseases. For example, C57BL/6 mice are usually resistant to collagen-induced arthritis but develop severe disease in the absence of TRAIL expression. ${ }^{12}$ Accordingly, gene transfer of TRAIL-encoding adenoviruses was able to significantly ameliorate clinical signs of arthritis. ${ }^{30}$ This beneficial effect of TRAIL gene therapy was most likely due to the induction of apoptosis in activated $\mathrm{T}$ cells and synoviocytes. Thus, these different studies illustrate that TRAIL has an important immunoregulatory role in the pathogenesis of rheumatoid arthritis, and also demonstrates the potential of TRAIL-based therapies.

The regulatory role of TRAIL appears not to be limited to experimental rheumatoid arthritis, but extends to other models of experimental autoimmune diseases. TRAIL expression is upregulated in pancreatic islets during the development of diabetes in nonobese diabetic (NOD) mice, ${ }^{31}$ a disease that is dependent on the infiltration and activation of autoreactive $\mathrm{T}$ lymphocytes. Similar to rheumatoid arthritis, neutralization of TRAIL by administration of soluble TRAIL receptor exacerbates the onset of diabetes development. In another model of autoimmune diabetes (streptozodin-induced diabetes), disease progression is more severe in TRAILdeficient than in TRAIL-competent mice. ${ }^{32}$ However, much alike FasL, TRAIL appears to have not only a protective regulatory role but may be potentially also involved in the actual tissue destruction process during diabetes. Human $\beta$ cells express TRAIL receptors and sensitivity to TRAILinduced apoptosis can be amplified by inhibition of protein synthesis or removal of TRAIL-R3. ${ }^{33}$ Finally, TRAIL has also been shown to mediate a regulatory function during the pathogenesis of experimental allergic encephalomyelitis (EAE), ${ }^{34}$ an animal model for multiple sclerosis in humans. Apart from this impressive regulatory potential of TRAIL in the regulation of autoreactive lymphocytes, it cannot be excluded that TRAIL, similar to FasL, also participates in the direct tissue destruction observed in most autoimmune dieases. Thus, dependent on time, dose and location of TRAIL expression, it may exert a beneficial as well as destructive potential.

One of the obvious questions rising from these observations is how TRAIL regulates immune responses and diseasepromoting immune cells. The regulatory role of TRAIL may not necessarily always be related to its apoptosis-inducing activity. For example, in the diabetes model, TRAIL has been proposed to inhibit the proliferation of diabetogenic $T$ cells by suppressing the production of IL-2 and subsequently inhibiting cell cycle progression, which could be compensated by exogenous addition of IL-2. In line with this notion are previous reports showing that TRAIL inhibits DNA synthesis in human autoreactive $T$ cells and prevents progression from $\mathrm{G} 1$ to $\mathrm{S}$ phase, without inducing apoptosis. ${ }^{35}$

Apart from these direct effects of TRAIL on autoreactive T cells, TRAIL may regulate autoimmunity also at the level of antigen-presenting cells. As mentioned above, DC may become sensitive to TRAIL-induced apoptosis under certain conditions, and thus reduced antigen presentation will result in reduced $\mathrm{T}$-cell activation and downregulation of the (auto-) immune response. ${ }^{4,5}$ It is well possible that both direct effects of TRAIL on autoreactive $T$ cells or indirect inhibition via effects on antigen-presenting cells are involved in the regulation of autoimmune diseases by TRAIL.

\section{TRAIL: a ligand or a receptor?}

Most recently, another surprising aspect of TRAIL biology has been described. Our current understanding of how members of the TNF family mediate their activities is through binding to their cognate receptors and thereby transducing activating or inhibitory signals. Recent evidence, however, demonstrates that transmembrane forms of TNF-related molecules may not only act as ligands, but instead also function as receptors and transduce signals via their cytoplasmic tails. This 'reverse signaling' has been shown for FasL, TNF $\alpha$, CD27L, CD30L, CD40L, OX-40L and also TRAIL (reviewed in Janssen et $\left.a .^{36}\right)$. In $\mathrm{T}$ cells reverse signaling via FasL and TRAIL appears to enhance cellular activation, leading to better proliferation and cytokine production. Most interestingly, while many of these molecules and in particular FasL contain signaling or binding motifs in their cytoplasmic domains, for example, phosphorylation sites or SH3-binding domains, ${ }^{36,37}$ TRAIL has only a very small cytoplasmic tail devoid of any recognizable signaling motifs. It is therefore currently completely unclear how the cytoplasmic tail of TRAIL can mediate this costimulatory effect. Since most studies have been using soluble TRAIL-R2 in order to induce reverse signaling in T cells, ${ }^{38,39}$ it is possible that the effect of soluble TRAIL-R2 on enhanced proliferation and cytokine production is actually not mediated by reverse signals, but through blockade of TRAIL 
interaction with its receptor and thus abolishment of negative regulatory signals. Further studies using a non-receptorinteracting mutant of TRAIL must show the real nature of and the importance of reverse signals mediated by the TRAIL cytoplasmic domain on T-cell activation.

\section{Conclusions and future directions}

In summary, while TRAIL has a strong potential in the induction of tumor cell apoptosis and tumor therapy, its effect on nontransformed and, in particular, immune cells appears to be equally important in the regulation of various physiological and pathophysiological processes (Figure 1b). The availability of TRAIL-deficient mice or neutralizing antibodies and soluble receptors, as well as reliable tools to assess TRAIL and TRAIL receptor expression in human tissue samples, has allowed and will allow to develop a more detailed picture on the role of TRAIL in immune regulation and tissue damage, opening a whole new field of novel therapeutic interventions.

\section{Acknowledgements}

We thank the Brunner lab and Andreas Villunger for helpful discussions. Work discussed in this review was supported by grants from the Swiss National Science Foundation, Oncosuisse, the Bernese Cancer League and the Novartis Foundation to TB.

1. Locksley RM et al. (2001) Cell 104: 487-501

2. Ursini-Siegel J et al. (2002) J. Immunol. 169: 5505-5513

3. Kayagaki $\mathrm{N}$ et al. (2002) Cell Immunol. 219: 82-91

4. Leverkus M et al. (2000) Blood 96: 2628-2631
5. Hayakawa $Y$ et al. (2004) J. Immunol. 172: 123-129

6. Simon HU (2003) Immunol. Rev. 193: 101-110

7. Renshaw SA et al. (2003) J. Immunol. 170: 1027-1033

8. Ludwig AT et al. (2004) Cancer Res. 64: 3386-3390

9. Simon AK et al. (2001) Proc. Natl. Acad. Sci. USA 98: 5158-5163

10. Wiley SR et al. (1995) Immunity 3: 673-682

11. Newton $\mathrm{K}$ et al. (2000) EMBO J. 19: 931-941

12. Lamhamedi-Cherradi SE et al. (2003) Nat. Immunol. 4: 255-260

13. Brunner T et al. (1995) Nature 373: 441-444

14. Cretney E et al. (2003) J. Exp. Med. 198: 491-496

15. Walsh CM et al. (1998) Immunity 8: 439-449

16. Corazza $\mathrm{N}$ et al. (2004) Cell Death Differ. in press

17. Strasser A et al. (1995) EMBO J. 14: 6136-6147

18. Bouillet $P$ et al. (2002) Nature 415: $922-926$

19. Lin $Y$ et al. (2000) Mol. Cell. Biol. 20: 6638-6645

20. Harris CA et al. (2001) J. Biol. Chem. 276: 37754-37760 Epub 32001 Aug 37758

21. Putcha GV et al. (2003) Neuron 38: 899-914

22. Brunner T et al. (2003) Semin. Immunol. 15: 167-176

23. Griffith TS et al. (1995) Science 270: 1189-1192

24. Lee $\mathrm{HO}$ et al. (2002) J. Immunol. 169: 4739-4744

25. Strebel A et al. (2002) Int. J. Cancer 100: 627-634

26. Phillips TA et al. (1999) J. Immunol. 162: 6053-6059

27. Nagata $S$ et al. (1995) Immunol. Today 16: 39-43

28. Cretney E et al. (2002) J. Immunol. 168: 1356-1361

29. Sedger LM et al. (2002) Eur. J. Immunol. 32: 2246-2254

30. Liu Z et al. (2003) J. Clin. Invest. 112: 1332-1341

31. Mi QS et al. (2003) Diabetes 52: 1967-1975

32. Lamhamedi-Cherradi SE et al. (2003) Diabetes 52: 2274-2278

33. Ou D et al. (2002) Diabetologia 45: 1678-1688 Epub 2002 Oct 1623

34. Suter T et al. (2003) Eur. J. Immunol. 33: 2998-3006

35. Lunemann JD et al. (2002) J. Immunol. 168: 4881-4888

36. Janssen $O$ et al. (2003) Cell Death Differ. 10: 1215-1225

37. Watts AD et al. (1999) EMBO J. 18: 2119-2126

38. Chou AH et al. (2001) J. Immunol. 167: 1347-1352

39. Chen NJ et al. (2001) J. Immunol. 166: 270-276 